\title{
Myocardial substrate after cardiac resynchronization therapy and the risk of ventricular arrhythmias
}

\author{
Saurabh Malhotra, MD, MPH, FASNC ${ }^{a}$ \\ a Division of Cardiovascular Medicine, Clinical and Translation Research Center, Jacob School of \\ Medicine and Biomedical Sciences at the University at Buffalo, Buffalo, NY
}

Received Mar 9, 2016; accepted Mar 10, 2016

doi: 10.1007/s12350-016-0474-7

\section{See related article, pp. 1282-1288}

Myocardial remodeling is a central feature of failing hearts. It can take several forms, and the most easily recognizable ones are electrical remodeling (wide QRS complex with or without left bundle branch block) and mechanical remodeling (dilated and hypofunctioning left ventricle). This remodeling results in disorganized left ventricular (LV) contraction, which further promotes heart failure (HF) progression and has been shown to be an independent predictor of adverse cardiac events. $^{1-3}$ Patients with myocardial remodeling and symptomatic HF that is refractory to medical therapy have been shown to derive symptomatic and mortality benefit from cardiac resynchronization therapy (CRT).,5 This benefit has been largely thought to be secondary to reduced interventricular conduction delay, reduction in LV size, and consequently improved LV contractility. ${ }^{6}$ The Comparison of Medical Therapy, Pacing and Defibrillation in Heart Failure (COMPANION) trial tested the efficacy of CRT-pacemaker and CRT-defibrillator with medical therapy alone. The trial reported that both CRT-pacemaker and CRT-defibrillator provided significantly better survival and reduced hospitalizations when compared with medical therapy alone. ${ }^{5,7}$ Though not specifically studied, the logical advantage of a CRT-defibrillator would be arrhythmia

Reprint requests: Saurabh Malhotra, MD, MPH, FASNC, Division of Cardiovascular Medicine, Clinical and Translation Research Center, Jacob School of Medicine and Biomedical Sciences at the University at Buffalo, 875 Ellicott Street, Suite 7030, Buffalo, NY 14221; drsmalhotra@hotmail.com,smalhot@buffalo.edu

J Nucl Cardiol 2017;24:1289-91.

1071-3581/\$34.00

Copyright (C) 2016 American Society of Nuclear Cardiology. prevention and CRT-pacemaker seemed to offer a similar level of survival, suggesting an anti-arrhythmic effect of CRT alone.

Since then other studies have reported a potential anti-arrhythmic benefit from CRT. Ermis et al prospectively followed 18 patients undergoing an upgrade of a defibrillator to a CRT-defibrillator, for arrhythmic events. ${ }^{8}$ Prior to an upgrade to CRT-defibrillator, $72 \%$ of the patients experienced an arrhythmic outcome as opposed to only $11 \%$ following the upgrade, suggesting a role of CRT in ameliorating ventricular arrhythmias in patients with HF. In another study, 107 patient with CRT-defibrillator were prospectively followed for arrhythmic events after elective generator change. ${ }^{9}$ Occurrences of arrhythmic events before and after generator change were recorded and were studied in relation to CRT response and an ongoing need for a defibrillator (defined as a LV ejection fraction $<40 \%$ ). This study showed that, at the time of the generator change, $87 \%$ of the CRT responders were free of arrhythmic events compared to $70 \%$ of CRT non-responders. Following an elective generator change, only $5 \%$ of those without an ongoing need for a defibrillator experienced arrhythmic events, when compared to $72 \%$ with an ongoing need for a defibrillator. Although both these studies imply reverse remodeling after CRT to confer protection against ventricular arrhythmia, this was not specifically evaluated. A recent sub-analysis of the Multicenter Automatic Defibrillator Implantation Trial-Cardiac Resynchronization Therapy (MADITCRT) trial specifically assessed the role of CRT-defibrillator in preventing arrhythmic outcomes. ${ }^{10}$ In this analysis, 1783 patients belonging to the MADIT-CRT study were stratified by LV ejection fraction of $\leq$ or $>30 \%$, and the occurrence of fast ventricular arrhythmias was determined in both these groups. Patients with LV ejection fraction $\leq 30 \%$ had a higher rate of ventricular arrhythmia when compared to those with LV 
ejection fraction $>30 \%$. Among those with a lower ejection fraction, CRT-defibrillator showed a greater reduction in the occurrence of ventricular arrhythmias when compared to ICD alone. Among those with a higher ejection fraction, this benefit from CRT-defibrillator was not seen, probably due to the low overall event rate. Although this sub-analysis of the MADITCRT trial and prior smaller studies underscore a potential anti-arrhythmic benefit from CRT-defibrillator, whether this anti-arrhythmic protection is due to reverse LV remodeling from CRT was not studied.

In this issue of the journal, Chiang et al examined the relationship between cardiac reverse remodeling after CRT with ventricular arrhythmia. ${ }^{11}$ The authors conducted a cross-sectional study of 41 patients who had received a clinically indicated biventricular $(\mathrm{BiV})$ pacemaker and had $>99 \%$ BiV pacing after 12 months following device implant. All patients underwent a single resting SPECT at approximately 12 months after initiation of CRT, from which indices of scar, LV function, and dyssynchrony were assessed. All patients also had a transthoracic echocardiogram performed at approximately 12 months after CRT from which mechanical reverse remodeling (MRR) was determined by comparing to the pre-CRT echocardiogram. In addition, change in intrinsic QRS duration with CRT was also assessed in all, by measuring QRS duration with CRT-on and off. The change in QRS duration was considered to be indicative of electrical reverse remodeling (ERR).

During an average follow-up of 1.4 years after gated SPECT, 19 patients experienced arrhythmic events (13 sustained ventricular tachycardia, 2 nonsustained ventricular tachycardia, and 6 ventricular fibrillation). On a multivariate cox regression analysis, MRR (i.e., change in LV ejection fraction by echocardiography), ERR (i.e., change in QRS duration before and after CRT), QRS duration with CRT-on (BiV pacing), LV ejection fraction on SPECT, and phase standard deviation (PSD) on phase analysis of SPECT images were independent predictors of arrhythmic events. Receiver operating curve (ROC) analysis identified optimal cutoff for each of these independent predictors of arrhythmic events: change in LV ejection fraction on echocardiography $\leq 7$ (AUC 0.85), change in QRS duration with CRT $\leq 7 \mathrm{~ms}$ (AUC 0.78), QRS duration with CRT-on $\geq 121 \mathrm{~ms}$ (AUC 0.68), LV ejection fraction on SPECT $\leq 30 \%$ (AUC 0.76), and PSD $\geq 45.6^{\circ}$ (AUC 0.74). Both changes in QRS duration after CRT and the QRS duration with CRT-on had the highest sensitivity (94\%) for predicting arrhythmic events. A critical observation from this study is the association of the magnitude of dyssynchrony after CRT (assessed by PSD) with ventricular arrhythmia. PSD had the highest specificity (84\%) for predicting arrhythmic events, among all the other significant variables. However, this study is limited by the lack of dyssynchrony assessment prior to CRT, and thus a relationship between change in dyssynchrony with CRT and arrhythmic events cannot be studied, in a fashion similar to that reported from another MADIT-CRT sub-analysis. ${ }^{12}$ Furthermore, the assessment of remodeling, function, scar, and dyssynchrony from more than one imaging modality makes the application of such an approach challenging in contemporary clinical practice.

Nonetheless, the results of this study highlight the relationship between MRR and ERR, after 1 year of CRT, with arrhythmic outcomes. This essentially implies that a lack of or inadequate improvement in the myocardial substrate with CRT is predictive of arrhythmic events and provides a physiological rationale for the prior studies reporting a reduction in arrhythmic events after CRT. Myocardial scar is an important contributor to LV dysfunction in HF patients and has been shown to predict arrhythmic events. ${ }^{13}$ However, in this study of patients with CRT and $\mathrm{BiV}$ pacing for at least 1 year, myocardial scar on SPECT was not an independent predictor of arrhythmic events. Myocardial scar likely exerts its pro-arrhythmic effect in more than one way-providing a focus of electrical vulnerability and also by promoting remodeling (measured as change in LV volume, ejection fraction, and QRS duration). Although the magnitude of myocardial scar will not change following CRT, it has been shown to predict CRT responders, in conjunction with other variables. In a prospective study of 44 patients, Friehling et al reported the presence of baseline dyssynchrony, myocardial scar $<40 \%$ of the LV myocardium, and LV lead concordance with the site of latest activation as independent predictors of acute improvement in LV synchrony after CRT and composite clinical outcomes (inclusive of ventricular arrhythmias). ${ }^{14}$ The report from Friehling et al and the current study by Chiang et al collectively suggest that both myocardial substrate prior to CRT and the change in this substrate with CRT are predictive arrhythmic events. Furthermore, the myocardial substrate that prevents reverse remodeling with CRT also seems to be the substrate that promotes arrhythmogenesis. This fact can have profound clinical implications, such as withholding of defibrillator implantation in those who are expected to improve with CRT or deactivation of the defibrillator function once improvement in myocardial substrate has been documented. Such an approach may have a potential of improving cost effectiveness of cardiac device therapies in HF and will also reduce the morbidity associated with inappropriate defibrillator therapy. ${ }^{15}$ On the contrary, those who are unlikely to improve with CRT, based on 
pre-CRT assessment of myocardial substrate, or those who did not improve after continuous BiV pacing should be considered at a high risk for arrhythmic events and continue to receive a defibrillator, as they do in contemporary clinical practice. Whether the acute change in myocardial substrate after CRT translates into sustained reverse remodeling, and whether both provide a similar prediction for arrhythmogenesis, needs to be systematically evaluated.

Another important aspect of this report by Chiang et $\mathrm{al}^{11}$ is the use of a risk factor-based model, incorporating several imaging findings, to predict those at the highest risk of arrhythmic events. Using the independent predictors of ventricular arrhythmia from their study, the authors developed a risk prediction model based on the number of these independent predictors: $\leq 2,3-4$, and 5 risk factors. On Kaplan-Meier survival analysis for time to ventricular arrhythmia, an increasing number of these independent predictors were associated with a worsening survival ( 2 risk factors vs 3-4 risk factors vs 5 risk factors; $P<0.001$ ). The annualized rate of arrhythmic events increased from approximately $6 \%$ among those with 2 risk factors to $35 \%$ and $53 \%$ among those with 34 and 5 risk factors, respectively. Use of a risk prediction model will understandably have a low sensitivity for predicting arrhythmic events; however, it offers a high specificity and a high negative predictive value to identify those at the lowest risk. This has been suggested in multiple prior studies utilizing imaging and biomarker-based risk prediction tools to predict arrhythmic events in various $\mathrm{HF}$ populations, ${ }^{16}$ including a gated SPECT-based model of LV ejection fraction, myocardial scar. and dyssynchrony. ${ }^{17}$ Although these risk prediction tools show great promise, they need to be prospectively validated in clinical studies.

\section{References}

1. Fauchier L, Marie O, Casset-Senon D, Babuty D, Cosnay P, Fauchier JP. Interventricular and intraventricular dyssynchrony in idiopathic dilated cardiomyopathy. A prognostic study with fourier phase analysis of radionuclide angioscintigraphy. J Am Coll Cardiol 2002;40:2022-30.

2. Bader H, Garrigue S, Lafitte S, Reuter S, Jaïs P, Haïssaguerre M, et al. Intra-left ventricular electromechanical asynchrony. A new independent predictor of severe cardiac events in heart failure patients. J Am Coll Cardiol 2004;43:248-56.

3. Cho GY, Song JK, Park WJ, Han SW, Choi SH, Doo YC, et al. Mechanical dyssynchrony assessed by tissue Doppler imaging is a powerful predictor of mortality in congestive heart failure with normal QRS duration. J Am Coll Cardiol 2005;46:2237-43.

4. Cleland JG, Daubert JC, Erdmann E, Freemantle N, Gras D, Kappenberger L, et al. The effect of cardiac resynchronization on morbidity and mortality in heart failure. $\mathrm{N}$ Engl $\mathrm{J}$ Med 2005;352:1539-49.

5. Bristow MR, Saxon LA, Boehmer J, Krueger S, Kass DA, De Marco T, et al. Cardiac-resynchronization therapy with or without an implantable defibrillator in advanced chronic heart failure. New Engl J Med 2004;350:2140-50.

6. Solomon SD, Foster E, Bourgoun M, Shah A, Viloria E, Brown $\mathrm{MW}$, et al. Effect of cardiac resynchronization therapy on reverse remodeling and relation to outcome: Multicenter automatic defibrillator implantation trial: Cardiac resynchronization therapy. Circulation 2010;122:985-92.

7. Anand IS, Carson P, Galle E, Song R, Boehmer J, Ghali JK, et al. Cardiac resynchronization therapy reduces the risk of hospitalizations in patients with advanced heart failure: Results from the Comparison of Medical Therapy, Pacing and Defibrillation in Heart Failure (COMPANION) trial. Circulation 2009;119:969-77.

8. Ermis C, Lurie KG, Zhu AX, Collins J, Vanheel L, Sakaguchi S, et al. Biventricular implantable cardioverter defibrillators improve survival compared with biventricular pacing alone in patients with severe left ventricular dysfunction. J Cardiovasc Electrophysiol 2004;15:862-6.

9. Sebag FA, Lellouche N, Chen Z, Tritar A, O'NEILL MD, Gill J, et al. Positive response to cardiac resynchronization therapy reduces arrhythmic events after elective generator change in patients with primary prevention CRT-D. J Cardiovasc Electrophysiol 2014;25:1368-75.

10. Kutyifa V, Moss AJ, Solomon SD, McNitt S, Aktas MK, Barsheshet A, et al. Reduced risk of life-threatening ventricular tachyarrhythmias with cardiac resynchronization therapy: Relationship to left ventricular ejection fraction. Eur J Heart Fail 2015; 17:971-8.

11. Chiang KF, Hung GU, Tsai SC, Cheng CM, Chang YC, Lin WY, et al. Impact of cardiac reverse remodeling after cardiac resynchronization therapy assessed by myocardial perfusion imaging on ventricular arrhythmia. J Nucl Cardiol 2016. doi:10.1007/s12350016-0447-x.

12. Kutyifa V, Pouleur AC, Knappe D, Al-Ahmad A, Gibinski M, Wang PJ, et al. Dyssynchrony and the risk of ventricular arrhythmias. JACC Cardiovasc Imaging 2013;6:432-44.

13. Morishima I, Sone T, Tsuboi H, Mukawa H, Uesugi M, Morikawa $\mathrm{S}$, et al. Risk stratification of patients with prior myocardial infarction and advanced left ventricular dysfunction by gated myocardial perfusion SPECT imaging. J Nucl Cardiol 2008; 15:631-7.

14. Friehling M, Chen J, Saba S, Bazaz R, Schwartzman D, Adelstein $\mathrm{EC}$, et al. A prospective pilot study to evaluate the relationship between acute change in left ventricular synchrony after cardiac resynchronization therapy and patient outcome using a single-injection gated SPECT protocol. Circ Cardiovasc Imaging 2011;4: 532-9.

15. Daubert JP, Zareba W, Cannom DS, McNitt S, Rosero SZ, Wang $\mathrm{P}$, et al. Inappropriate implantable cardioverter-defibrillator shocks in MADIT II: Frequency, mechanisms, predictors, and survival impact. J Am Coll Cardiol 2008;51:1357-65.

16. Malhotra S, Canty JM, Jr. Life-threatening ventricular arrhythmias: Current role of imaging in diagnosis and risk assessment. $\mathbf{J}$ Nucl Cardiol 2016.

17. Hou PN, Tsai SC, Lin WY, Cheng CM, Chiang KF, Chang YC, et al. Relationship of quantitative parameters of myocardial perfusion SPECT and ventricular arrhythmia in patients receiving cardiac resynchronization therapy. Ann Nucl Med 2015;29:772-8. 